\title{
Videostroboscopy in Laryngopharyngeal Reflux Disorder
}

\author{
${ }^{1}$ S Raghunandhan, ${ }^{1}$ Jawahar Nagasundaram, ${ }^{1}$ Kiran Natarajan, ${ }^{2}$ Srividya Prashanth, ${ }^{1}$ Mohan Kameswaran \\ ${ }^{1}$ Consultant ENT Surgeons, Madras ENT Research Foundation, Chennai, Tamil Nadu, India \\ ${ }^{2}$ Registrar, Madras ENT Research Foundation, Chennai, Tamil Nadu, India
}

Correspondence: Mohan Kameswaran, Madras ENT Research Foundation, No. 1, 1st Cross Street, Off 2nd Main Road, Raja Annamalaipuram, Chennai, Tamil Nadu, India, e-mail: merfmk30@yahoo.com

\begin{abstract}
Introduction: Acid reflux disease of the larynx has been established as a separate clinical entity in the present day, requiring the nuances of a skilled laryngologist for its early diagnosis and appropriate management. Clinical presentations of laryngopharyngeal reflux disorder may vary from very subtle to mammoth proportions, ranging across a panorama of features like chronic laryngitis, indolent laryngeal ulcers or granulomas, onto life-threatening glottic/sub-glottic stenosis and laryngeal malignancies very rarely. With the advent of videostroboscopy, laryngologists have now acquired a 'cutting-edge' sensitive tool to look into the intricacies of the larynx and thereby study the pathological effects of laryngopharyngeal reflux in great detail.

Objective: To assess the efficacy of videostroboscopy with voice analysis, as a diagnostic and prognostic tool in a cohort of patients with laryngopharyngeal reflux disorder.

Study method: This prospective study was conducted over a two-year period, among 50 patients with hoarseness of voice and features of reflux laryngitis, who underwent videostroboscopy with voice analysis at initial diagnosis and at routine follow-up, to objectively study the response and outcome of anti-reflux therapy on the larynx.

Observation and result: Videostroboscopy provided a vivid picture of the changes in larynx due to chronic acid reflux and proved efficacious in precisely judging the treatment responses.

Conclusion: Our study reflects the recent concept highlighted in world literature that videostroboscopy is an ideal, sensitive tool for early diagnosis and in prognosis of acid induced laryngeal disorder.
\end{abstract}

Keywords: Acid laryngitis, Gastroesophageal reflux disorder (GERD), Laryngopharyngeal reflux disorder (LPR), Videostroboscopy, Lingwave voice analysis, Anti-reflux therapy, Proton pump inhibitors (PPI).

\section{INTRODUCTION}

Laryngopharyngeal reflux disorder (LPR) is a unique entity, gaining phenomenal significance in recent literature, due to its rising incidence worldwide. For long, it had prevailed as a relatively unknown component in the spectrum of acid peptic disorders, being in the purview of gastroenterologists and mostly going unnoticed under the confluence of symptoms, broadly termed as gastroesophageal reflux disorders (GERD). Until recent times, investigations and treatment modalities for gastroesophageal reflux disorder primarily focused on the lower esophageal sphincter, which was attributed to be the primary focus for inducing the reflux symptoms. Present literature has shifted its focus onto the upper esophageal sphincter, which in reality is predominantly responsible for most patient's complaints.

Laryngopharynx is a dynamic, pivotal region safeguarding the ai rway from the caustic influence of gastrointestinal juices. $M$ alfunction of the laryngopharyngeal sphincter results in chronic insult to the larynx, resulting in a spectrum of laryngeal lesions. A large number of patients with voice problems, when evaluated by a sensitive tool such as videostroboscopy, have been found to have a significant contribution due to laryngopharyngeal reflux. A Iso on the contrary, many established cases of acid peptic disease have subtle, subclinical manifestations in the larynx which remain masked by the predominant GE RD features, until growing onto severe proportions. Hence, early diagnosis and appropriate treatment of l aryngopharyngeal reflux prevails as a vital factor in the management of voice disorders.

\section{Study Methodology}

This prospective study was devised to objectively analyze the significance of laryngopharyngeal reflux as an etiological factor in patients presenting with hoarseness of voice and to assess their subsequent improvement in laryngeal symptoms with antireflux therapy. The principal aim of our study was to assess the efficacy of videostroboscopy with voice analysis in the diagnosis and follow-up of patients with laryngopharyngeal reflux disorder. This case study was conducted at the Department of V oice and Reflux Disorders, M ER F - Chennai, from February 2007 to J anuary 2009, over a 2-year period.

A total of 50 adult patients were selected for the study, based upon the inclusion and exclusion criteria formulated as in Table 1. The voice changes like breathiness, hoarseness or vocal strain needed to be progressive or persistent for a minimum period of 3 weeks, despite preliminary treatment with oral 
Videostroboscopy in Laryngopharyngeal Reflux Disorder

Table 1: Inclusion and exclusion criteria

\begin{tabular}{ll}
\hline Inclusion criteria & Exclusion criteria \\
\hline Group I: Adults diagnosed with GERD on regular & - Professional voice users \\
treatment and recent onset of change in voice persistent & - h/o vocal abuse \\
for 3 weeks & $-\mathrm{h} / \mathrm{o} \mathrm{previous} \mathrm{laryngeal} \mathrm{surgery} \mathrm{or} \mathrm{trauma}$ \\
Group II: Adults without prior h/o GERD & $-\mathrm{h} / \mathrm{o}$ uncontrolled endocrinal or metabolic disturbances \\
and recent onset of voice change & - h/o organic and/or neurological lesions involving the larynx, \\
persistent for 3 weeks, associated with & laryngopharynx or esophagus. \\
symptoms of burning sensation in throat, & \\
dry hacking cough and dyspepsia. & \\
\hline
\end{tabular}

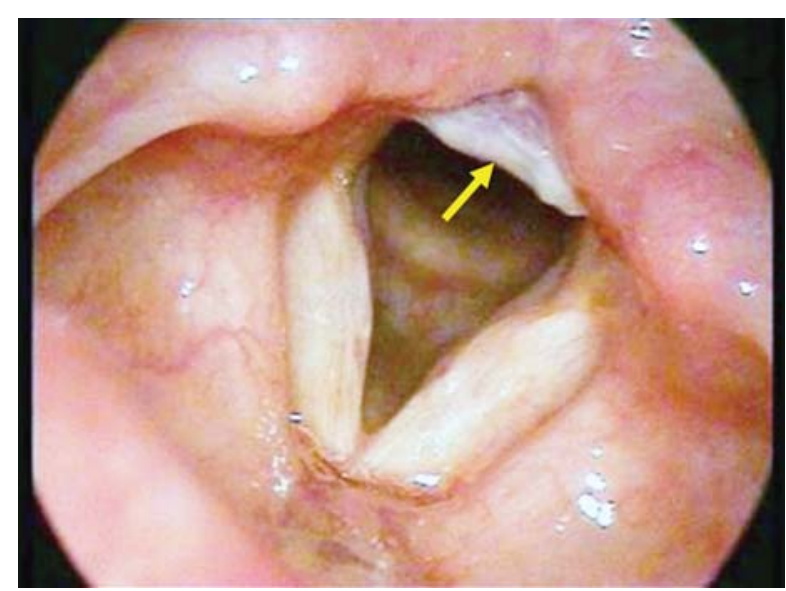

Fig. 1: Videostroboscopic picture showing classical features of posterior laryngitis, vocal fold thickening and arytenoid edema with interarytenoid banding (arrow) in a patient with laryngopharyngeal reflux disorder

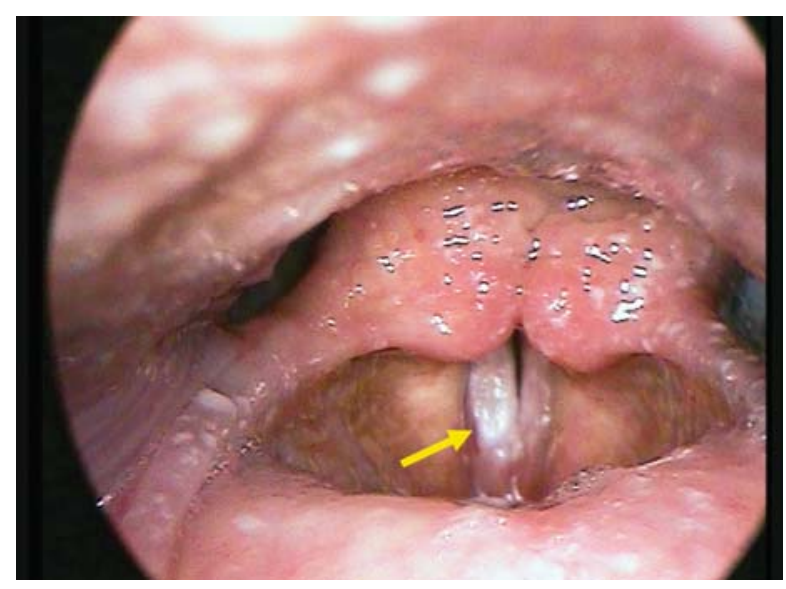

Fig. 2: Videostroboscopic picture showing gross acid reflux and right vocal fold hypertrophy with leukoplakia (arrow) and posterior glottic chink

medications and voice rest. A fter obtaining informed consent for participation in the study, patients included in the cohort underwent a comprehensive otorhinolaryngological examination including a detailed laryngeal assessment by videostroboscopy ('A tmos' M ediaStrobo M ES M edien Elektronik Software, Germany, Version 2.0) incorporated with voice analysis software ('lingW aves' LingCom G mbH Forchheim, Germany, V ersion 2.4) at the commencement of the study in

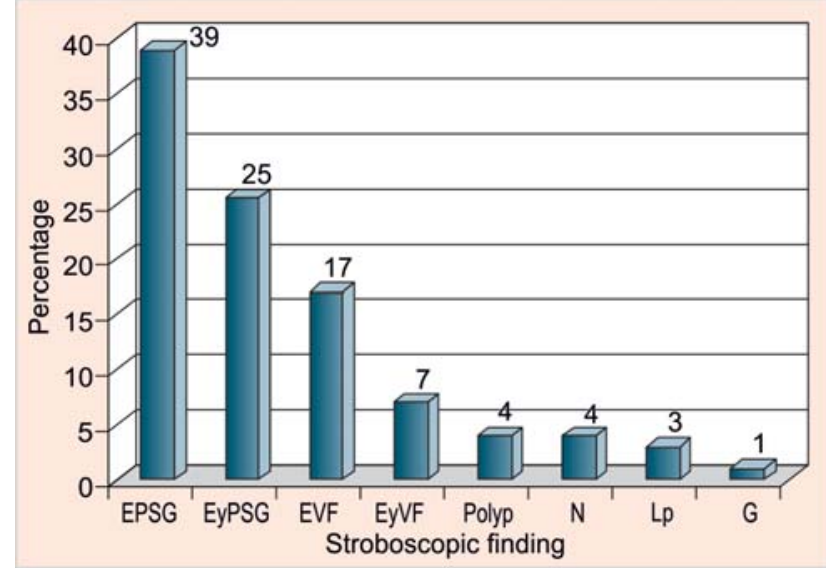

Fig. 3: Spectrum of laryngeal pathology in LPR: EPSG: Edema of posterior supraglottis; EyPSG: Erythema of posterior supraglottis; EVF : Edema of vocal folds; EyVF: Erythema of vocal folds; P: Polyp, $\mathrm{N}$ : Nodule; Lp: Leukoplakia, G: Contact granuloma

order to document their laryngeal and vocal findings (Figs 1 and 2). The varied spectrum of manifestations of $L P R$ on videostroboscopic examination was recorded and documented for further follow-up (Fig. 3).

A fter videostroboscopic confirmation of the diagnosis of LPR, all patients were started on a standardized 12 weeks regimen of antireflux medications (proton pump inhibitors and prokinetic agents) and were followed up for 6 months, with sequential videostroboscopic assessment and voice analysis at regular intervals of 1,3 and 6 months during which they were also symptomatically graded based on the improvement of voice and relief from reflux features as per Reflux Symptom Index Score ${ }^{1}$ (Fig. 4).

B oth the clinical response and videostroboscopic eval uation during the course of treatment were meticulously documented until the completion of the study period. The efficacy of videostroboscopy as a sensitive diagnostic and prognostic tool, in these patients with laryngopharyngeal reflux was assessed by sequentially monitoring the changes taking place in the larynx, in response to antireflux therapy by LPR index/reflux finding scores. ${ }^{1}$ Symptomatic relief from LPR and subjective improvement in voice while on treatment were compared with objective signs of remission of LPR, as documented by videostroboscopy and voice analysis. 


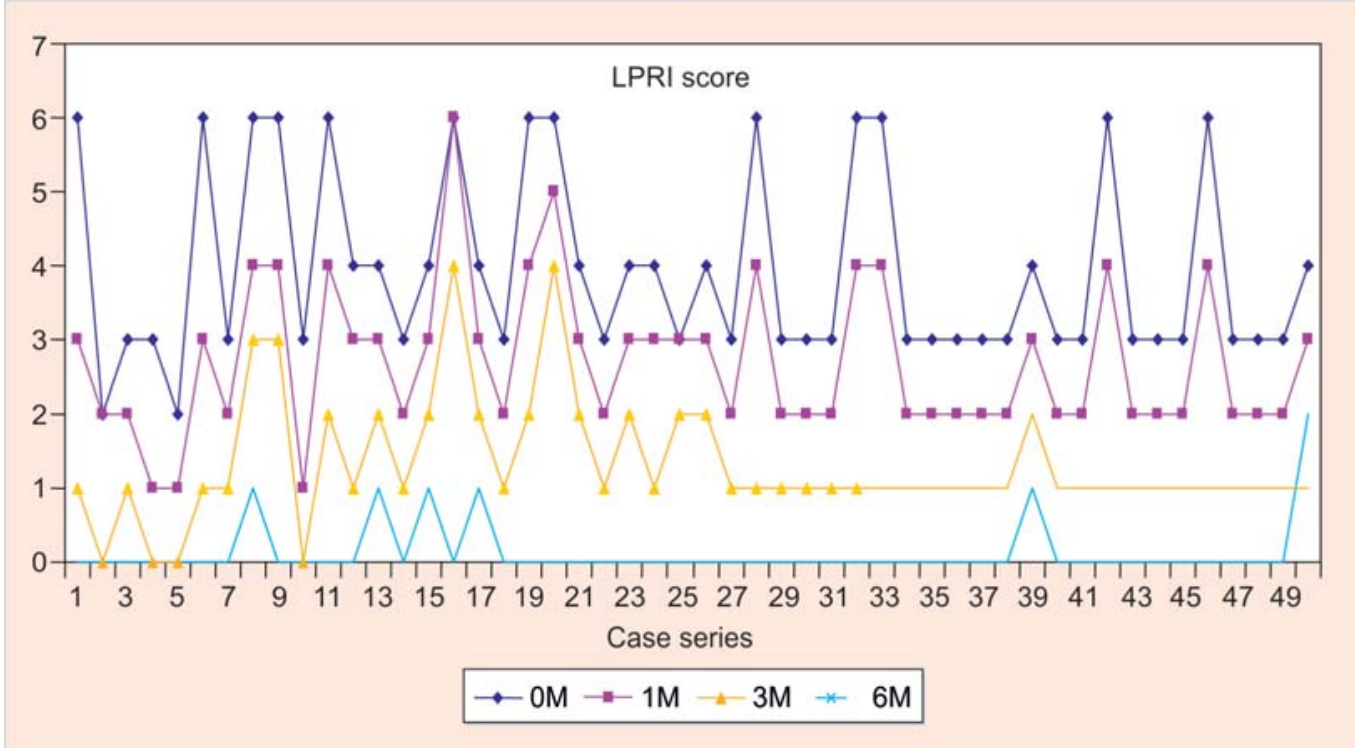

Fig. 4: Comparison of LPR symptom index score at 0, 1, 3 and 6 months follow-up

[1] Fle Edit Andysis Window Options Hel

[.

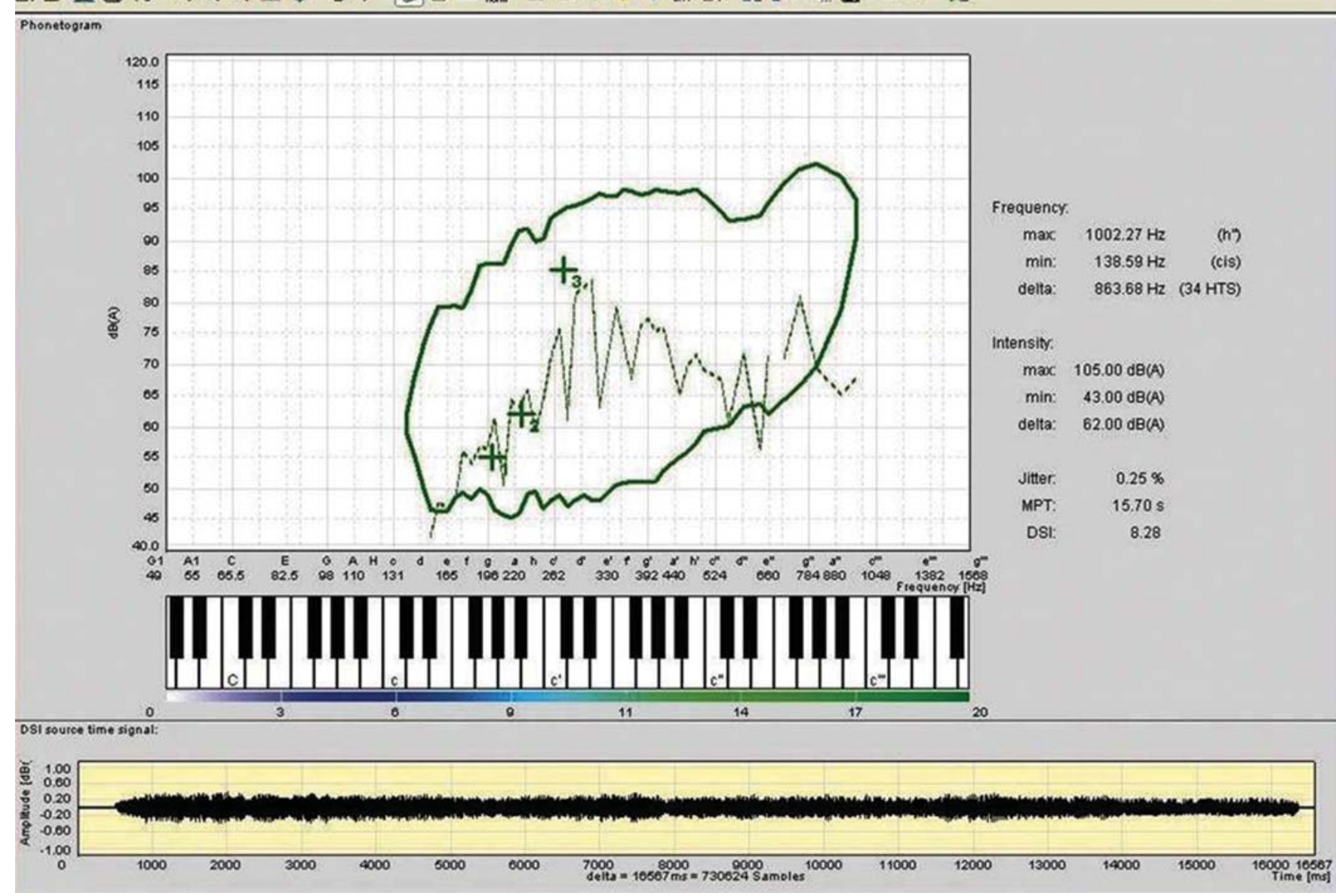

Fig. 5: Lingwave voice analysis for a patient with dysphonia induced by LPR (chart depicts a phonautogram with dysphonia severity index: The intensity of the patient's formant is displayed as a broken line. Three crosses mark the speaking range profile for normal, soft and loud voice performance. The DSI provides a one-dimensional value for the categorization and evaluation of voice quality)

Qualitative analysis of glottic function was done, as per videostroboscopy findings, with special focus on parameters like amplitude, symmetry, periodicity and regularity of vocal fold movements, presence of complete glottal closure or a phonatory gap/glottic chink, nature of mucosal wave patterns and presence of supra or sub-glottic pathologies. Perceptual voice analysis was done as per the standardized GRBAS 4point scale, as described by Hirano in 1981. The voice was marked on a scal e from 0 to 4 , on the parameters of $G$ rade $-G$, roughness $-R$, breathiness $-B, A$ sthenia $-A$ and Strain $-S$. 
The objective measure of vocal quality was analyzed by a phonetogram with harmonic to noise ratio (HNR) and dysphonia severity index $(D S I)^{2}$ as described by Professor FL W uyts, through a formula of the weighted values of "highest frequency", "lowest intensity in the phonautogram", "maximal phonation time (M PT)" and the relation of V oice Jitter to M PT (Fig. 5).

\section{OBSERVATIONS AND RESULTS}

A mong the 50 patients in our study group, LPR disorder was most common in the age group of 40 to 50 years, with a small predominance in females (54\%) over males (46\%). Edema of posterior supraglottis, erythema of posterior supraglottis and edema of vocal folds were the three most common findings on initial stroboscopic examination. Findings such as erythema of vocal folds, polyp, nodule, leukoplakia and granuloma were al so observed to a lesser extent. M ajority of our patients (90\%) responded well to the treatment with proton pump inhibitors and prokinetic agents given over 12 weeks period and had a normal stroboscopic picture with normal voice quality by the end of antireflux therapy.

A t initial assessment, voice analysis in patients with LPR show ed an exacerbation of the breathiness and strain component on the GRBAS scale. There was marked elevation in the dysphonia severity index (DSI) along with abnormal Jitter (fundamental frequency perturbation) and Shimmer (fundamental amplitude perturbation) parameters. The maximum phonatory time (M PT) was markedly reduced and the phonatory frequency ' $F_{0}$ ' was al tered. Significant improvement in all these parameters could be well documented by the end of 4 weeks of antireflux therapy in $68 \%$ of our study group, while rest of the patients required further time between 3 to 6 months for achieving normal voice quality.

The mean dysphonia severity index (DSI) scores [(group I = 20 (range 14-27) and group II = 19 (range 5-24)], were comparable between the two study groups prior to initiation of anti-reflux therapy. The overall post-therapy averagemean DSI score for both groups was 9.0 ( $p<0.05$ as compared with the baselinenormative). Theinitial mean average j itter scores were comparable (group I $=0.8$, group II =0.5), as were the mean averageShimmer scores (group I $=3.1$, group II =2.8). Thefinal perceptual voice analysis for both groups showed very similar results and the combined mean average J itter score was 0.2 ( $p$ $<0.02$ ) and mean average Shimmer score was $1.3(p<0.01)$ at the completion of the anti-reflux therapy. The mean average maximum phonatory time (M PT) of both groups at initiation of therapy was 12.3 seconds, which improved to 19.7 seconds at the end of antireflux therapy. Faster resolution of all vocal parameters into the normal range was recorded in group II as compared to group I, during the course of follow-up.

The final set of voice analysis performed at 6 months showed normal parameters in $90 \%$ of the combined study group. In $10 \%$ of the patients (i.e. 5 patients), LPR had induced significant changes in the glottis, refractory to anti-reflux therapy and hence persistent alteration in the voice quality. These L PR-induced persistent lesions like leukoplakia, granul oma and keratosis had to be laserized with KTP/532 Laser using a precise microlaryngeal surgical technique and subsequent improvement in voice quality was eventually achieved.

\section{DISCUSSION}

Early research in 1995 by Delahunty and Cherry proved that inflammation, ulceration and formation of granulation tissue on the vocal fold mucosa followed extended exposure to gastric material. Further reports by U lualp et al ${ }^{3}$ suggested that chronic acid reflux patients present with increased interarytenoid or posterior glottic inflammation and erythema, hypertrophy of the posterior commissure (cobblestoning/interarytenoid banding) and granulation tissue formation in severe cases, which was best diagnosed by videostroboscopy.

Proton pump inhibitors (PPI) are particularly effective in improving the healing rate in patients with laryngopharyngeal reflux. H2-blockers are often effective in treating GERD patients by "turning the acid down", which is not sufficient treatment for many patients with LPR. The acid needs to be virtually "turned off", since the larynx is far more susceptible to injury from the acidic reflux than the esophagus. L aryngeal mucosa lacks the protective mechanism and barrier that the esophageal mucosa has to prevent damage from acid and pepsin exposure. This includes the lack of acid buffering capability, absence of normal peristalsis to clear the refluxate and the presence of a thinner, more susceptible and sensitive mucosal lining in the larynx. ${ }^{3}$

Optimal treatment of LPR is possible only with complete cessation of acid-pepsin injury to the larynx. For this reason, a minimum of twice-daily dosing of PPI is recommended for treatment of $L P R$. The recommended duration of initial therapy with PPI should be a minimum of three months. ${ }^{3} \mathrm{~A}$ II the patients in our prospective study were treated with proton pump inhibitors (PPI) for a minimum of 3 months and followed up with periodic videostroboscopic assessment of the larynx with voice analysis for objective evaluation of the response to antireflux therapy.

The natural course of a LPR-induced laryngeal lesion tends to be indolent and long standing in many patients and it may turn virulent or premalignant rarely in a few cases, if left untreated. Q adeer et $\mathrm{al}^{4}$ in a general population based randomized study of 72 patients noted that the mean average age of these patients with LPR disease was 50 years and it was more common in females $(60 \%)$. He reported that the most common symptoms in his study group were sore throat (40\%), followed by hoarseness of voice $(30 \%)$ and chronic cough (20\%). Our study cohort followed almost the same trends in age incidence and LPR symptoms as reported by the $\mathrm{Q}$ adeer et al study. 
B ook et al ${ }^{5}$ reported videostroboscopy to be an efficacious tool in the early diagnosis of gastroesophageal reflux disorders influencing the larynx. He reported arytenoid erythema (97.5\%), vocal cord erythema/edema $(95.7 \%)$, posterior commissure hypertrophy (94.9\%), and arytenoid edema (94.0\%) as the most common stroboscopic findings among these patients. B eaver et al ${ }^{6}$ noted that edema and erythema of supraglottis, glottis and subglottis appeared to be of equal involvement in laryngopharyngeal reflux disease and appropriate antireflux therapy provided full recovery of all parts of larynx from acid insult, which was remarkably documented by serial videostroboscopy at regular follow-up. B ranski RC et al ${ }^{7}$ emphasized that serial endoscopic imaging with a sensitive tool such as the videostroboscope, proved vital as a prognosticator in judging patient responses to antireflux medications and voice therapy.

Cesari et $\mathrm{al}^{8}$ performed a prospective study in 62 patients with hoarseness of voice, who had no clinical symptoms of LPR and no history of vocal abuse. He derived a significant correlation between LPR and dysphonia, based on objective investigative modalities, including 24 hours pH monitoring, video laryngopharyngoscopy and voice analysis. He concluded that a direct correlation exists between dysphonia and LPR, which gradual ly progresses over time due to contributory factors like arytenoid muscles dysfunction and chronic vocal fatigue resulting from the stress of glottic insufficiency compensation. $V$ ideostroboscopic assessment periodically done during antireflux therapy in our patients, followed the same path to recovery as per C esari's study, wherein these contributory factors were abolished with treatment and normal vocal function could be ultimately obtained.

Voice analysis of patients with LPR has been recently described in world literature. ${ }^{9,10}$ The consensus arrived from the various studies is that LPR patients have significantly altered frequency perturbation when compared to normal subjects, and their vocal manifestation can be indolent and subclinical for a period of time, until when the hoarseness becomes symptomatically evident. The jitter scores significantly correlate with the LPR reflux symptom index and the acoustical parameters of $J$ itter, Shimmer, HNR and DSI improve well within 1 to 3 months of antireflux therapy and reach their normal values at the completion of treatment. In a few refractory cases, a combined modality of intensive voice therapy along with prolonged antireflux treatment may be necessary. ${ }^{9,10}$

\section{CONCLUSION}

V ideostroboscopic assessment of patients suspected to have laryngopharyngeal reflux gives us a vivid picture of the ultramicroscopic changes which occur early within the larynx due to acid insult. A sequential follow-up of such patients under treatment with videostroboscopic assessment and voice analysis at periodic intervals, provide us a clear picture of the response to antireflux therapy. The results of our study is comparable with present world literature which supports the efficacy of videostroboscopy as a sensitive predictor and a prognosticator in the management of patients with laryngopharyngeal reflux disorder.

\section{REFERENCES}

1. Belafsky PC, Postma GN, K oufman J A. V alidity and reliability of the reflux symptom index (RSI). J V oice 2002;16:274-77.

2. Selby JC, Gilbert HR, Lerman JW. Perceptual and acoustic evaluation of individuals with laryngopharyngeal reflux pre- and post-treatment. J V oice 2003;17(4): 557-70.

3. Ulualp SO, Toohill RJ, et al. Laryngopharyngeal reflux: State of the art diagnosis and treatment. Otolaryngol Clinics of $\mathrm{N}$ orth A merica Feb 2000:151-61.

4. Qadeer, M ohammed A, Swoger, Jason. Correlation between symptoms and laryngeal signs in laryngopharyngeal reflux: Laryngoscope 2005;115(11):1947-52.

5. Book DT, et al. Perspectives in laryngopharyngeal reflux: An international survey. Laryngoscope 2002;112:1399-1402.

6. Beaver M ary E, Stasney Richard C, W eitzel Erik, Stewart Miichael G, Donovan Donald T, Parke Robert B, Rodriguez $M$ argarita: Diagnosis of laryngopharyngeal reflux disease with digital imaging. Otolaryngol Head and Neck Surg 2003;128: 103-08.

7. Branski RC, Bhattacharya $\mathrm{N}$, Shapiro J. The reliability of assessment of endoscopic laryngeal findings of laryngopharyngeal reflux disease. Laryngoscope 2002;112:1019-21.

8. Cesari U, Galli J, Ricciardiello F, Cavaliere M, Galli V. Dysphonia and laryngopharyngeal reflux. A cta Otorhinolaryngol Ital 2004;24(1):13-19.

9. Oguz H, Tarhan E, Korkmaz M , Y ilmaz U, Safak M A, D emirci $M$. A coustic analysis findings in objective laryngopharyngeal reflux patients. J Voice 2007;21(2):203-10.

10. Jin J, Lee Y S, J eong SW, J eong JH, L ee SH, T ae K. Change of acoustic parameters before and after treatment in laryngopharyngeal reflux patients. L aryngoscope 2008;118(5):938-41. 